\title{
Comparisons of Force Measurement Methods for DBD Plasma Actuators in Quiescent Air
}

\author{
Alan R. Hoskinson* and Noah Hershkowitz ${ }^{\dagger}$ \\ University of Wisconsin-Madison \\ David E. Ashpis ${ }^{\ddagger}$ \\ NASA Glenn Research Center at Lewis Field, Cleveland, Ohio, USA
}

\begin{abstract}
We have performed measurements of the force induced by both single (one electrode insulated) and double (both electrodes insulated) dielectric barrier discharge plasma actuators in quiescent air. We have shown that, for single barrier actuators with cylindrical exposed electrodes, as the electrode diameter decrease the force efficiencies increase much faster than a previously reported linear trend. This behavior has been experimentally verified using two different measurement techniques: stagnation probe measurements of the induced flow velocity and direct measurement of the force using an electronic balance. Actuators with rectangular cross-section exposed electrodes do not show the same rapid increase at small thicknesses. We have also shown that the induced force is independent of the material used for the exposed electrode. The same techniques have shown that the induced force of a double barrier actuator increases with decreasing narrow electrode diameter.
\end{abstract}

\section{Introduction}

Tumerous groups have demonstrated that when dielectric barrier discharges (DBDs) are used to generate plasma on a flat surface, they can induce a flow velocity in the surrounding atmospheric pressure neutral gas. ${ }^{1-5}$ The effect generally increases when using a strongly asymmetric electrode configuration and when operating in air as opposed to nitrogen. ${ }^{6}$ The induced flow is believed to be similar to the "electric wind" phenomenon noted in corona discharges, ${ }^{7}$ caused by the collision of electric-field-accelerated charged particles colliding with neutral gas molecules. When the DBD is used for active flow control, it is commonly termed a plasma actuator.

Single barrier plasma actuators, such as the one diagrammed in figure 1, have been studied intensely both experimentally ${ }^{4,5,8-10}$ and computationally. ${ }^{7,11-13}$ Previously, Enloe et al. ${ }^{4}$ noted that decreasing the diameter of the exposed electrode of a single barrier actuator linearly increased the actuator force efficiency (force / power) without changing the discharge power or emitted light. Increasing the actuator force is highly desirable, but based on the linear extrapolation of Enloe's data only a limited benefit could be obtained by reducing the exposed electrode diameter. In this paper we show that for small diameter electrodes the induced force efficiency increases much faster than linearly with decreasing diameter.

Double barrier actuators, such as the one pictured in figure 2, have been much less widely studied than the single barrier geometry, in part due to the observation of lower induced velocities. ${ }^{14}$ We include them in this study as they have fewer possible sources of irregularities in the experiments. Double barrier actuators have no features above the surface to impede the induced air flow, and the electrodes are not in physical contact with the plasma so their material characteristics should not affect the discharge. Double barrier actuators

\footnotetext{
${ }^{*}$ Graduate Research Assistant, Engineering Physics, University of Wisconsin, Madison, Wisconsin 53706, AIAA Student Member

${ }^{\dagger}$ Irving Langmuir Professor, Engineering Physics, University of Wisconsin, Madison 53706

¥National Aeronautics and Space Administration, Glenn Research Center at Lewis Field, Cleveland, Ohio 44135, USA, AIAA Member
} 


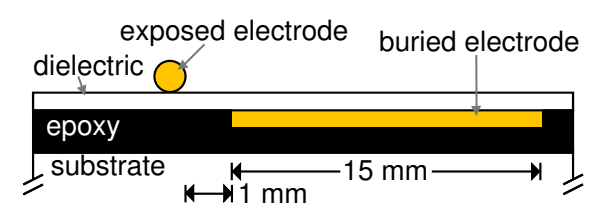

Figure 1. Single barrier actuator

show an increase in force efficiency with decreasing narrow electrode diameter, although the absolute forces are significantly smaller than those found for single barrier actuators.

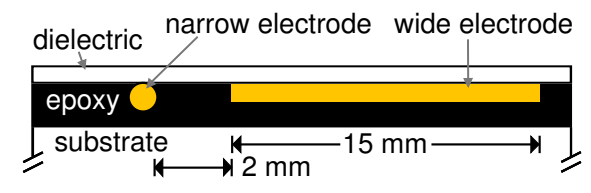

Figure 2. Double barrier actuator

The two actuator geometries considered in this work, single and double barrier, are described in figures 1 and 2 , respectively. The induced air flow moves from left to right in those figures. For all actuators used in this work, the dielectric layer was made from $0.25 \mathrm{~mm}$ thick polyester film, which had a dielectric constant of 3.3. An insulating epoxy filled gaps beneath the dielectric layer, and had a dielectric constant of 3.8. The substrate layer was used for mechanical mounting, and is not believed to be involved in device performance.

Buried or wide electrodes were made from $40 \mu \mathrm{m}$ thick copper tape, while exposed and narrow electrodes were made from a variety of materials. All electrodes spanned $60 \mathrm{~mm}$ in the direction perpendicular to the plane of figures 1 and 2. For all single barrier actuators a $1 \mathrm{~mm}$ horizontal gap separated the closest edges of the exposed and buried electrodes. For all double barrier actuators the horizontal gap between narrow and wide electrodes was $2 \mathrm{~mm}$. This wider gap was necessary to avoid electric breakdown of the epoxy at higher applied voltages.

For all measurements presented here, the actuators were driven by symmetric triangle waveforms oscillating at $1 \mathrm{kHz}$. The applied voltages were supplied by a Trek 20/20B high voltage amplifier and passed through a two-stage low-pass filter to remove RF frequency noise on the signal. For single barrier actuators the high voltage was connected to the exposed electrode, while for double barrier actuators it was connected to the narrow electrode. The buried or wide electrodes were grounded through the measurements capacitor as described in section II.C.

Some of the results discussed in this conference paper have been recently published by Hoskinson et al. ${ }^{15}$

\section{Measurement Techniques}

This work used two independent techniques to measure the force efficiency of each plasma actuator. We concern ourselves only with the force acting parallel to the dielectric surface, which is the $x$ direction in figure 3. Data from a stagnation probe approximately determined the velocity distribution near each actuator, which was then converted to the force of the actuator on the air as described below. This technique provided excellent sensitivity for small forces. A more direct measurement of the force, with limited sensitivity, was made with an electronic balance. The discharge power was determined by integrating the change in the charge on a measurement capacitor. All measurements were performed in quiescent atmospheric pressure air.

\section{A. Stagnation Probe Measurements}

We measured approximate air velocities using a small, movable stagnation probe, as shown in figure 3. To eliminate ambient air currents these measurements were conducted in a sealed cylindrical chamber $17 \mathrm{~cm}$ tall and $30 \mathrm{~cm}$ in diameter, with the actuator placed near the center of the chamber. The stagnation probe consisted of a glass capillary tube $0.4 \mathrm{~mm}$ in inner diameter with $0.2 \mathrm{~mm}$ thick walls. A Dwyer model 607-01 differential pressure transmitter measured the pressure difference between the probe and a reference 
port on the bottom of the chamber. For velocity measurements the stagnation probe was pointed into the induced flow: the $-x$ direction in figure 3 . All measurements reflect time-averaged values. The measurement technique is similar to that used by Pons et al. ${ }^{9}$

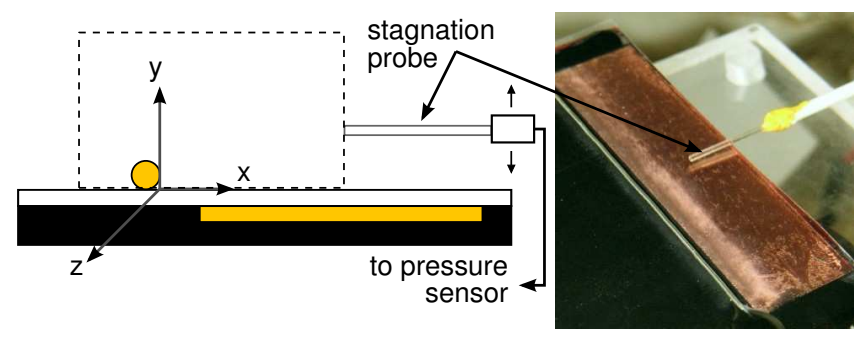

Figure 3. Orientation of stagnation probe for flow measurements and the control volume used in the analysis

To make comparisons between the stagnation probe and balance measurements, we must convert the measured pressures into forces, and then force efficiencies. The conversion to force follows, and is similar to the analysis performed by Baughn et al. ${ }^{16}$ To analyze the system, we consider the rectangular control volume of figure 3 . The control volume borders the dielectric surface and includes the entire plasma volume. The momentum balance equation for the $x$ direction is

$$
\frac{F_{\text {plasma }}}{L_{z}}+\frac{F_{\text {shear }}}{L_{z}}=\underbrace{\rho \int_{\text {right }} v_{x}^{2} d y}_{\text {I }}+\underbrace{\rho \int_{\text {top }} v_{x} v_{y} d x}_{\text {II }}-\underbrace{\rho \int_{\text {left }} v_{x}^{2} d y}_{\text {III }}+\underbrace{\int_{\text {right }} p d y}_{\text {IV }}-\underbrace{\int_{\text {left }} p d y}_{\mathrm{V}} .
$$

The integral subscripts indicate over which side of the control volume the integral is performed. $L_{z}$ is a unit length in the $z$ direction, $\rho$ is the density of air, and $p$ is the static pressure. Previous measurements by Enloe et al. ${ }^{6}$ indicated that air density fluctuations near the actuator plasma are generally smaller than $2 \%$ of the background density. These fluctuations are insignificant relative to other sources measurement errors. The included surface forces are static pressure forces on left and right surfaces and shear acting between the dielectric surface and the induced flow. We note that the shear force acts to slow the $+x$-directed flow and so is always negative. The only included body force is the force of the plasma on the air.

To limit the amount of data required to infer the force of each actuator, we have simplified equation (1) by making several assumptions. The validity and effects of these assumptions are estimated in section III.C. First, we assume that the static pressure is constant and equal to the reference pressure, $p_{\text {ref }}$, at the chamber bottom. As the chamber was open to room air prior to being sealed off to reduce measurement noise, $p_{\text {ref }}$ is approximately equal to atmospheric pressure for these measurements. The assumption of constant static pressure allows us to cancel terms IV and V against each other in equation (1). The assumption also permits us to use

$$
v_{x} \approx \sqrt{2\left(p_{0}-p_{\text {ref }}\right) / \rho}
$$

to convert from the measured stagnation pressure, $p_{0}$, to the air flow velocity. The assumption of a constant static pressure was implicitly made by Pons et al. ${ }^{9}$ in their measurements near a single barrier actuator, as they used an equation like (2) to determine the velocity. In addition to the assumption of constant static pressure, we neglect the momentum flux into the control volume through the left and top edges, setting terms II and III in equation (1) to zero.

Once these assumptions are made, equation (1) simplifies to

$$
\frac{F_{\text {plasma }}}{L_{z}}+\frac{F_{\text {shear }}}{L_{z}} \approx \rho \int_{\text {right }} v_{x}^{2} d y .
$$

To simplify the analysis, we use a skewed Gaussian,

$$
v_{\text {fit }}=v_{0} \sqrt{y} \exp \left[-\left(\frac{y-y_{0}}{L}\right)^{2}\right],
$$

fit to the measured data when evaluating equation (3). An example fit is shown in figure 4. 


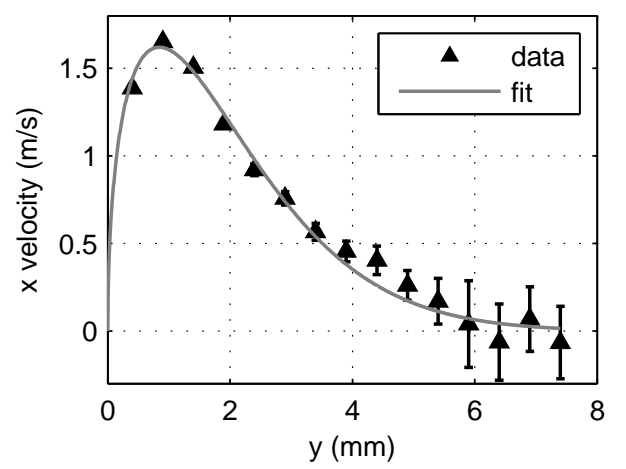

Figure 4. Skewed Gaussian fit to the velocities measured near a single barrier actuator with $d=0.11 \mathrm{~mm}$

Equation (3) is equivalent to the result of Baughn et al. ${ }^{16}$ with zero background velocity. That study measured the absolute plasma force and so placed the shear force on the right-hand side of the equation. Here we are interested in the net induced forces in order to compare to the forces measured directly with an electronic balance. All stagnation pressures used for force estimates were made at $x=8 \mathrm{~mm}$. Our calculations therefore only include the shear force up to $8 \mathrm{~mm}$, while the balance measurements include the shear force out to the edge of the dielectric at approximately $16 \mathrm{~mm}$. We estimate the effect of this discrepancy in section III.C.

\section{B. Electronic Balance Measurements}

To obtain a more direct measurement of the plasma actuator-induced force, we have used an Ohaus model PA313 electronic balance to determine the force. The balance has $1 \mathrm{mg}\left(10^{-5} \mathrm{~N}\right)$ resolution. While this resolution is coarser than that allowed by the stagnation probe measurements, the balance measurement is significantly more direct. The force measurement was made by mounting an actuator on a stand with the air flow directed upwards. The downward reaction force on the actuator is then measured by the balance as a positive force. Measurements on the electronic balance were conducted inside an acrylic box to prevent room air currents from affecting the measurement.

The high voltage and ground connections were made to the actuator using light 32 AWG wire to eliminate any force caused by sagging wires. Two undesirable effects caused by the high voltage signal were noted and removed. Electromagnetic coupling between the high voltage line and the balance manifested itself as a spurious negative force until the balance was covered with a grounded copper mesh. An insulating platform fit through a hole in the mesh and provided a place on which to set the actuator stand. An electrostatic attraction between the high voltage wire and the copper mesh was minimized by making the actuator stand tall enough to keep the wire approximately $15 \mathrm{~cm}$ above the mesh, which tests showed reduced the attractive force to below the resolution limit of the balance. Figure 5 shows the balance and stand used in these measurements.

In order to make direct comparisons to the stagnation probe measurements, the balance data must be converted into a force per unit length. We did this by dividing the total measured force by the width of buried or wide electrode $(6 \mathrm{~cm})$, which approximates the plasma width. We estimate the plasma width variation from actuator to actuator to be on the order of $\pm 5 \%$, leading to an error the same order as the uncertainty in the force measurement itself.

\section{Power measurements and conditioning}

Force efficiencies were determined by dividing the forces, measured using the procedures described above, by the dissipated electrical power. We determined this power by placing a $0.10 \mu \mathrm{F}$ capacitor in the ground path of the actuator and measured the voltage across it. Provided the measurement capacitance is much larger than the capacitance of the actuators (which were generally smaller than $10 \mathrm{pF}$ ), the circuit characteristics are unaffected by its presence. The actuator current is found by taking the time derivative of the charge 


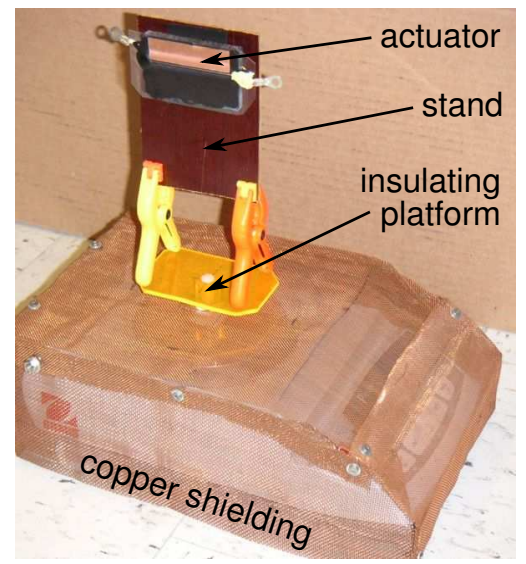

Figure 5. An actuator, actuator stand, insulating platform, and shielded balance used in direct force measurements

on the capacitor, and the time-averaged power determined by integrating the product of the applied voltage and the actuator current over an integer number of voltage cycles. ${ }^{9}$

Previous measurements have shown that actuator behavior exhibits run-to-run variation unless the discharge is operated for some time immediately prior to measurement. ${ }^{14,15}$ To ensure repeatability, all measurements presented here were recorded immediately after the actuators were run at the specified voltage level for at least $30 \mathrm{~min}$.

\section{Results and Discussion}

\section{A. Single Barrier Actuator Measurements}

We have conducted force and power measurements on single barrier actuators with narrow electrodes ranging from $0.03 \mathrm{~mm}$ to $1.0 \mathrm{~mm}$ in diameter, made from copper $(\mathrm{Cu})$, tungsten $(\mathrm{W})$, or stainless steel (SS). The electrical power used by each actuator has been measured using the method described in section II.C. The values of this power are relatively constant with changing electrode diameter, decreasing only slightly for actuators with exposed electrode diameters smaller than $0.2 \mathrm{~mm}$. The measured powers are shown in figure 6 , and are independent of the electrode material.

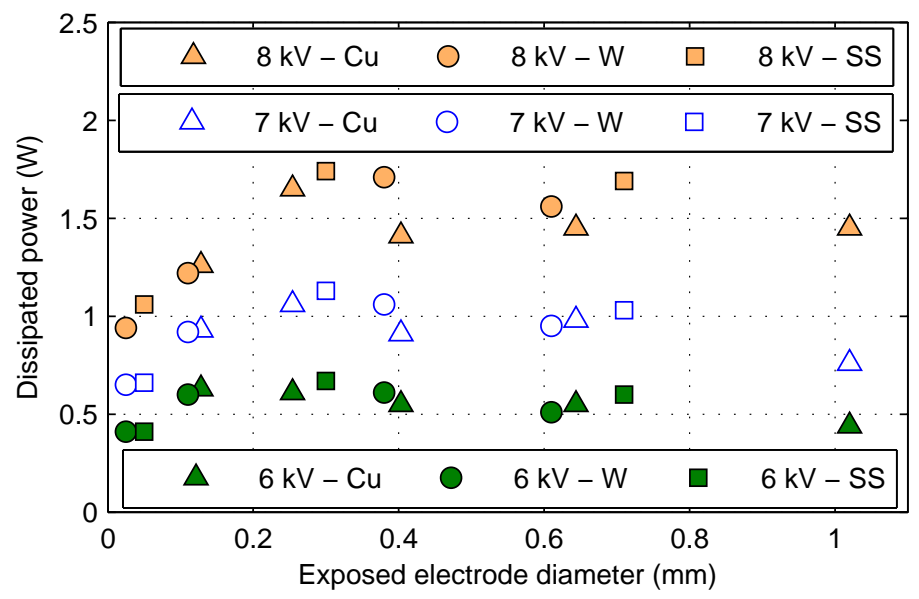

Figure 6. Time-averaged electrical powers dissipated by the single barrier actuators

Figure 7 shows the force efficiencies (force / power) per length inferred from the stagnation probe measurements, obtained using the methods described in sections II.A and II.C. The data indicate that, like with the discharge power, the material of the exposed electrode plays no role in determining the force efficiency. 
We also note that, in the range examined, the force efficiencies are essentially independent of the amplitude of the applied voltage waveform.

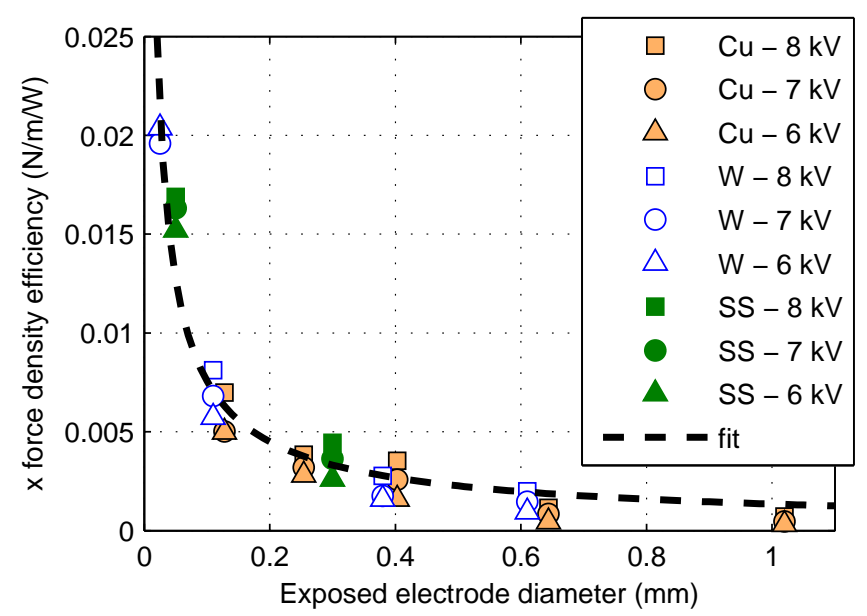

Figure 7. Time-averaged single barrier actuator-induced force efficiencies at three different voltage amplitudes, derived from stagnation probe measurements. The exposed electrodes of each actuator were composed of the indicated materials.

The stagnation probe-measured force efficiencies can be reasonably approximated as a function of the exposed electrode diameter using a power-law fit:

$$
f=A d^{-b} .
$$

$A$ and $b$ are free parameters, while $d$ is the electrode diameter. For the stagnation-probe data, we find $b=0.75 \pm 0.04$ This fit is also included in figure 7 .

We have also used the electronic balance method described in section II.B to directly measure the induced force of the actuators. The force efficiencies from these experiments are shown in figure 8 for the same amplitudes of the applied voltage used in the stagnation probe experiments. The balance measurements confirm the three major conclusions of the stagnation probe tests: the power law dependence of the induced force efficiencies on the diameter of the exposed electrode and the independence of the force efficiencies from both the material of the exposed electrode and the applied voltage amplitude. Here the power law fit found the same scaling parameter, within error, as the stagnation probe measurements, at $b=0.73 \pm 0.02$. The fit is shown in figure 8

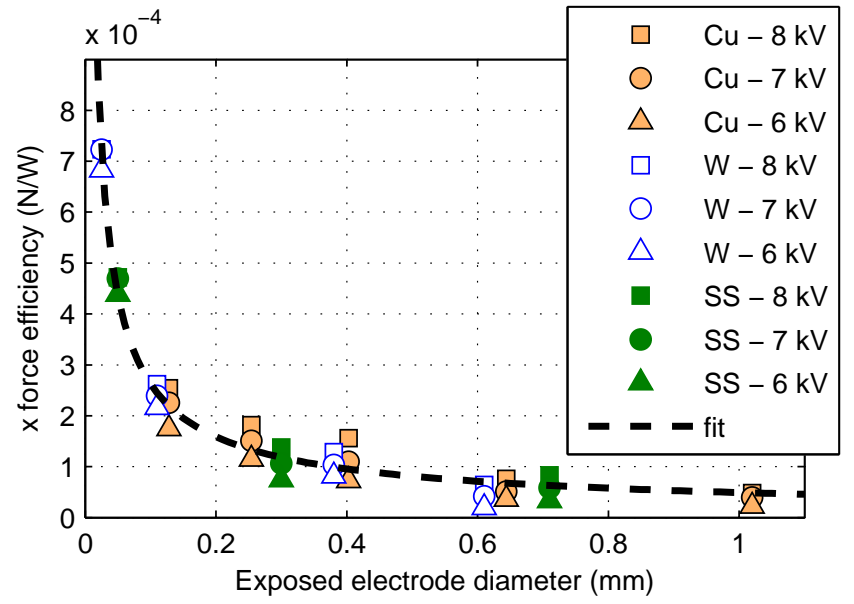

Figure 8. Time-averaged single barrier actuator-induced force efficiencies measured using the electronic balance. The exposed electrode of each actuator is composed of the indicated material.

It is likely that the major difference between the electrode materials is their differing secondary electron 
emission coefficients, although the literature regarding these for appropriate ions and energies is sparse. Based on simulation results, Boeuf et al. ${ }^{17}$ predicted that the value of the secondary electron emission coefficient of the electrode (which, in their model, was set equal to the coefficient for the dielectric) would have little effect on the force induced by the actuator, while being a strong predictor of dissipated power. Our data suggests that changing the secondary electron emission coefficient of just the exposed electrode has little effect on the induced force or on the dissipated power.

A study by Enloe et al. ${ }^{4}$ examined exposed electrode thicknesses down to approximately $0.1 \mathrm{~mm}$, with the smallest electrodes being rectangular rather than cylindrical, and noted a linear trend of increasing force efficiency with decreasing electrode thickness. Our measurements, in contrast, have shown a much faster increase as the electrode thickness is reduced. To examine this discrepancy, we constructed and tested several actuators with rectangular cross-section exposed electrodes. These stainless-steel electrodes were $10 \mathrm{~mm}$ long in the $x$ direction. The actuators had the same $1 \mathrm{~mm}$ horizontal spacing between electrodes as did those using cylindrical electrodes. The results of those measurements along with the same force data used in figure 8 are shown in figure 9. At present, only actuators with rectangular electrodes of $0.0125,0.025$, and $0.50 \mathrm{~mm}$ thicknesses have been tested.

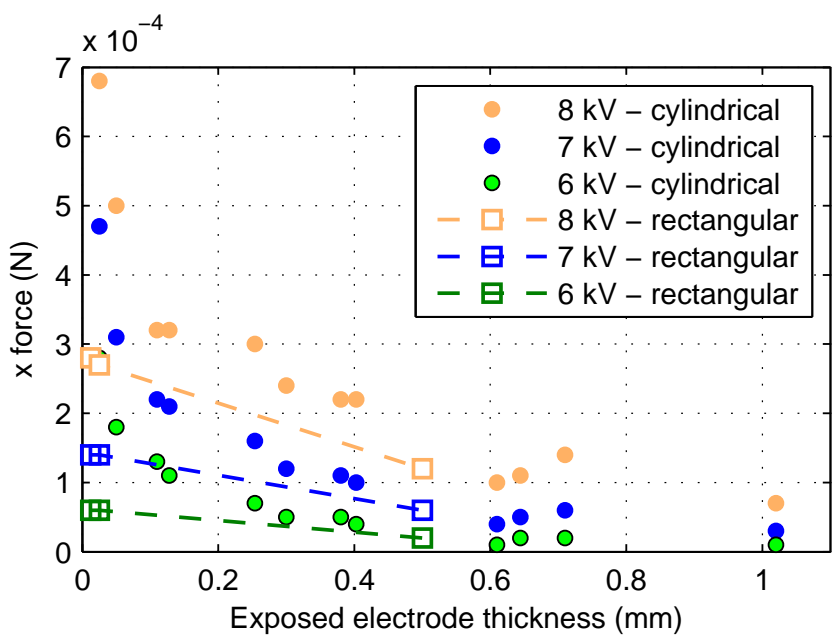

Figure 9. Time-averaged forces induced by single barrier actuators with rectangular and cylindrical exposed electrodes, measured using the electronic balance

The actuators with thin rectangular exposed electrodes induced forces significantly lower than those with cylindrical exposed electrodes of the same thickness. This effect likely explains the discrepancy between our results and those of Enloe et al. ${ }^{4}$ In our tests, the power dissipated by the actuators with rectangular electrodes was comparable at $0.50 \mathrm{~mm}$ and increased for the smaller thicknesses, in contrast to the behavior for cylindrical electrodes. Further testing is necessary to determine the range of thicknesses over which these differences between electrode geometries exist, as well as their cause.

\section{B. Double Barrier Measurements}

We have conducted the same force and power measurements on a series of double barrier actuators, described by figure 2. Double barrier actuators require higher applied voltages compared to single barrier actuators in order to generate plasma. The rates of current "spikes" are, however, much lower. The combination leads to slightly lower power use by double barrier actuators in the parameter ranges tested here. The measured powers are shown in figure 10. Power use increases roughly linearly as the narrow electrode diameter increases.

Similar to the behavior of single barrier actuators, the double barrier actuators induce larger force efficiencies when the electrode diameter, in this case of the narrow electrode, is reduced. Figure 11 shows the force efficiencies measured using the stagnation probe technique. The measured forces are smaller than those seen for single barrier actuators by roughly a factor of 5 . Actuators with narrow electrodes smaller than $0.1 \mathrm{~mm}$ in diameter tended to cause arcing through the dielectric layer, and so could not be studied.

Like the single barrier actuator force efficiencies, the data in figure 11 are well fit by the power law of 


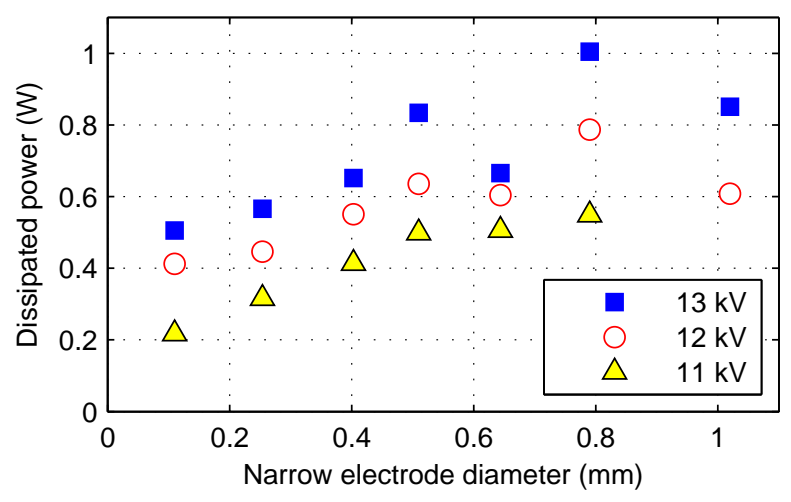

Figure 10. Time-averaged electrical power dissipated by double barrier actuators

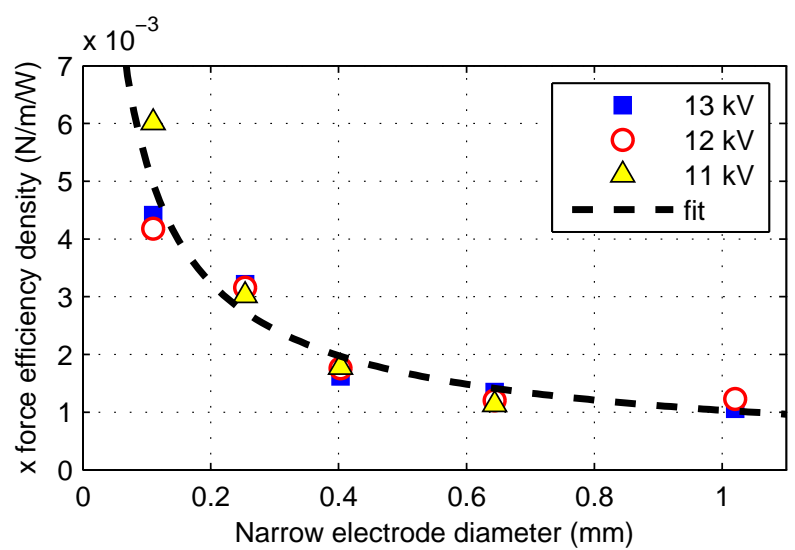

Figure 11. Time-averaged double barrier actuator-induced force efficiencies derived from stagnation probe measurements

equation (4). For these data we find a similar exponent of $b=0.71 \pm 0.07$, and the fit is also included in figure 11 .

Force efficiency measurements using the electronic balance, shown in figure 12, generally confirm the trend observed in the stagnation probe data, although the measured forces are small enough to approach the sensitivity limit of the balance. The fitted value of $b$ was identical, within error, to that seen for the stagnation probe data at $b=0.74 \pm 0.11$ The uncertainties in this fitting parameter are larger for the double barrier actuator data as the lower absolute force values cause relatively larger errors in our measured data.

\section{Comparison of Measurement Methods}

Nearly identical trends in the induced force efficiencies with varying electrode diameter have been observed using both a stagnation probe technique and an electronic balance. When the force densities are quantitatively compared, as in figure 13, the balance data are consistently lower by a factor of approximately two. We have determined that this difference is largely due to the assumptions made in section II.A to convert the measured stagnation pressures into force densities. We have made additional detailed measurements around one actuator (a single barrier actuator with a $0.11 \mathrm{~mm}$ diameter exposed electrode, driven by a $7 \mathrm{kV}$ amplitude voltage), and found that the removing most of our assumptions yields agreement between the stagnation probe and balance data.

We measured variations in both stagnation and static pressures at several $x$ locations, as shown in figure 14. Static pressures were determined using a $2.5 \mathrm{~mm}$ diameter alumina tube sealed at one end, with a $1 \mathrm{~mm}$ diameter hole drilled in the side $7 \mathrm{~mm}$ from the sealed end. The tube was oriented in the $-x$ direction, with the hole facing the $z$ direction.

The measurements confirm our assumption of constant static pressure in the region downstream $(+x$ 


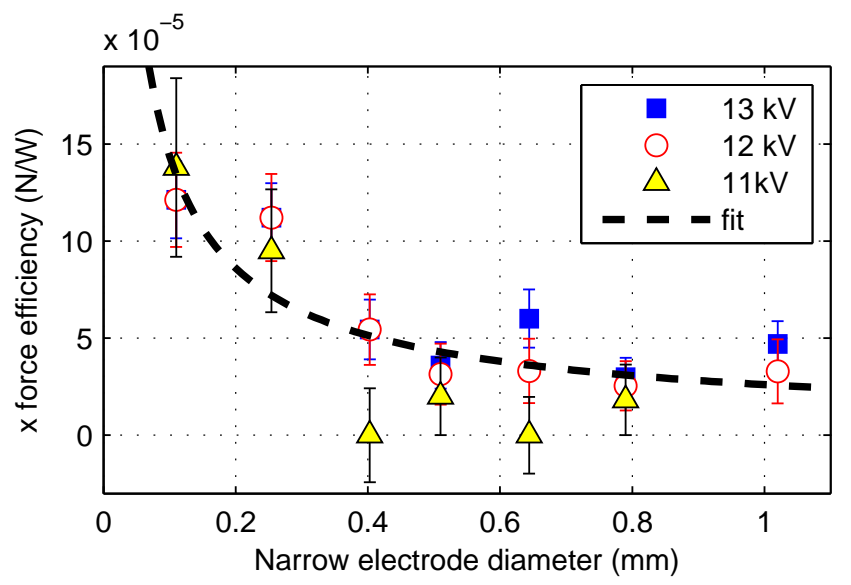

Figure 12. Time-averaged double barrier actuator-induced force efficiencies measured using the electronic balance, along with analytic fits to the data.

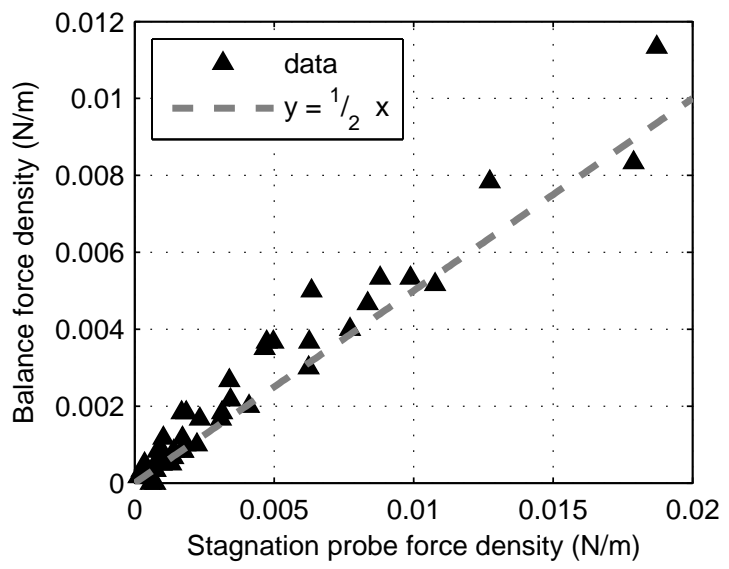

Figure 13. Comparison of force densities measured using the electronic balance and the stagnation probe technique using the assumptions of section II.A

direction) of the high voltage electrode, but show a small non-zero static pressure upstream. Our assumption of no momentum inflow through the left-hand edge of the control volume in figure 3 (that is, our neglect of term III in equation (1)) is seen to be incorrect. Although the flow velocity in this region is relatively small, it extends much further in the $y$ direction than does the narrow jet we observe on the right-hand edge of the control volume. After integrating, we see that term III is equal to $35 \%$ of the uncorrected force density, which was the largest correction to our original estimate. This estimate of term III in equation (1) itself may be slightly in error: the static probe was large enough to possibly disturb the gas flow in the region of measurement. Still, we believe including the term as measured is likely to be more accurate than continuing to neglect it completely.

Using the data of figure 14 we can evaluate the right-hand side of equation (1), except for term II, using the measured static pressure in place of the reference pressure in equation (2). Evaluating terms I and IV along the surface at $x=8 \mathrm{~mm}$ and terms III and $\mathrm{V}$ on the $x=-4 \mathrm{~mm}$ surface, we find a linear force density of $4.2 \times 10^{-3} \mathrm{~N} / \mathrm{m}$, or $68 \%$ of the original force estimate for these conditions. If we repeat the calculation and instead evaluate terms I and IV at $x=16 \mathrm{~mm}$, thereby including the shear force all the way to the actuator edge, we calculate a linear force density of $3.3 \times 10^{-3} \mathrm{~N} / \mathrm{m}$. This is $53 \%$ of the original estimate, and compares well to the balance-measured value of $3.7 \times 10^{-3} \mathrm{~N} / \mathrm{m}$. The significantly improved agreement between the two measurement techniques indicates that the assumptions made in section II.A are not strictly valid. 

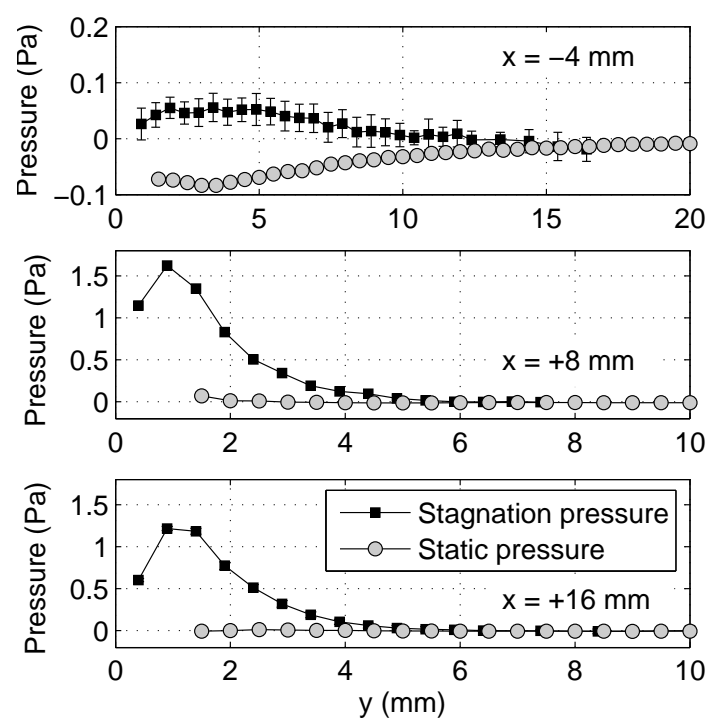

Figure 14. Stagnation and static pressures measured at $x=-4$ (top), +8 (middle), and +16 mm (bottom) for a single barrier actuator with $d=0.11 \mathrm{~m}$ driven at $7 \mathrm{kV}$

We have not attempted to estimate the effect of the flux of $x$ momentum through the top surface (term II in equation (1)), as we cannot accurately measure $v_{y}$ in that region. PIV measurements and calculations ${ }^{18,19}$ suggest that on the top edge of our control volume $v_{x}$ is much smaller than its values on the left- and righthand edges, and comparable to $v_{y}$. Provided these two quantities are small enough when integrated over the relevant edge, our neglect of term II would be reasonable. The relatively close agreement between the corrected stagnation probe data and the balance measurement also supports this conclusion.

\section{Conclusion}

We have observed faster-than-linear increases in the force efficiency of single barrier actuators as the exposed electrode diameter decreases. This behavior is well modeled by a simple power law fitting. Both forces and force efficiencies have been shown to be independent of the exposed electrode material. These behaviors have been confirmed using two independent techniques: direct measurement using an electronic balance and calculating the force from stagnation probe measurements. Simplifying assumptions used in calculating the induced forces from stagnation probe measurements tended to cause overestimations of the force. Reducing the actuator diameter to the extent possible suggests a simple way to significantly increase the forces and force efficiencies induced by these actuators, although the effect is much weaker if rectangular cross-section exposed electrodes are used. The same two measurement techniques have shown force efficiency growth as the narrow electrode diameter is reduced in double barrier actuators.

\section{Acknowledgments}

This work was supported by NASA under cooperative agreement NNX07AB84A and the Air Force Office of Scientific Research (AFOSR) under grant FA9550-07-1-0025. The authors thank Dr. Riccardo Bonazza for helpful discussions regarding the fluid dynamics calculations, and Dr. Jon Ahn for suggestions regarding our pressure measurement set-up.

\section{References}

\footnotetext{
${ }^{1}$ Roth, J. R., Sherman, D. M., and Wilkinsons, S. P., "Electrohydrodynamic flow control with a Glow-Discharge Surface Plasma," AIAA J., Vol. 38, No. 7, July 2000, pp. 1166-1172.

${ }^{2}$ Hultgren, L. S. and Ashpis, D. E., "Demonstration of separation delay with glow-discharge plasma actuators," 41 st AIAA Aerospace Sciences Meeting, Jan. 2003, 2003-1025.
} 
${ }^{3}$ Post, M. L. and Corke, T. C., "Separation control on high angle of attack airfoil using plasma actuators," AIAA J., Vol. 42, No. 11, Nov. 2004, pp. 2177-2184.

${ }^{4}$ Enloe, C. L., McLaughlin, T. E., VanDyken, R. D., Kachner, K. D., Jumper, E. J., Corke, T. C., Post, M., and Haddad, O., "Mechanisms and responses of a single dielectric barrier plasma actuator: Geometric effects," AIAA J., Vol. 42, No. 3, Mar. 2004, pp. 595-604.

${ }^{5}$ Opaits, D. F., Neretti, G., V., L. A., Zaidi, S., Shneider, M. N., and Miles, R. B., "Experimental investigation of DBD plasma actuators driven by repetitive high voltage nanosecond pulses with DC or low-frequency sinusoidal bias," 38th AIAA Plasmadynamics and Lasers Conference, June 2007, 2007-4532.

${ }^{6}$ Enloe, C. L., McLaughlin, T. E., and Font, G. I., "Parameterization of temporal structure in the single-dielectric-barrier aerodynamic plasma actuator," AIAA J., Vol. 44, No. 6, June 2006, pp. 1127-1136.

${ }^{7}$ Boeuf, J. P. and Pitchford, L. C., "Electrohydrodynamic force and aerodynamic flow acceleration in surface dielectric barrier discharge," J. Appl. Phys., Vol. 97, No. 103307, May 2005.

${ }^{8}$ Enloe, C. L., McLaughlin, T. E., VanDyken, R. D., Kachner, K. D., Jumper, E. J., and Corke, T. C., "Mechanisms and responses of a single dielectric barrier plasma actuator: Plasma morphology," AIAA J., Vol. 42, No. 3, 2004, pp. 589-594.

${ }^{9}$ Pons, J., Moreau, E., and Touchard, G., "Asymmetric surface dielectric barrier discharge in air at atmospheric pressure: Electrical properties and induced airflow characteristics," J. Phys. D, Vol. 38, Sep. 2005, pp. 3635-3642.

${ }^{10}$ Enloe, C. L., McLaughlin, T. E., Gregory, J. W., Medina, R. A., and Miller, W. S., "Surface Potential and Electric Field Structure in the Aerodynamic Plasma Actuator," 46th AIAA Aerospace Sciences Meeting, Jan. 2008, 2008-1103.

${ }^{11}$ Likhanskii, A. V., Shneider, M. N., Macheret, S. O., and Miles, R. B., "Modeling of interaction between weakly ionized near-surface plasmas and gas flow," 44th AIAA Aerospace Sciences Meeting, Jan. 2006, 2006-1204.

${ }^{12}$ Boeuf, J. P., Lagmich, Y., Unfer, T., Callegari, T., and Pitchford, L. C., "Electrohydrodynamic force in dielectric barrier discharge plasma actuators," J. Phys. D, Vol. 40, Jan. 2007, pp. 652-662.

${ }^{13}$ Likhanskii, A. V., Shneider, M. N., Macheret, S. O., and Miles, R. B., "Modeling of dielectric barrier discharge plasma actuators driven by repetitive nanosecond pulses," Phys. Plasmas, Vol. 14, No. 073501, July 2007.

${ }^{14}$ Hoskinson, A. R. and Hershkowitz, N., "Flow measurements and plasma simulations of double and single barrier DBD plasma actuators in quiescent air," 46th AIAA Aerospace Sciences Meeting, Jan. 2008, 2008-1370.

${ }^{15}$ Hoskinson, A. R., Hershkowitz, N., and Ashpis, D. E., "Force measurements of single and double barrier DBD plasma actuators in quiescent air," J. Phys. D, Vol. 41, Nov. 2008, pp. 245209.

${ }^{16}$ Baughn, J. W., Portoer, C. O., Peterson, B. L., McLaughlin, T. E., Enloe, C. L., Font, G. I., and Baird, C., "Momentum Transfer for an Aerodynamic Plasma Actuator with an Imposed Boundary Layer," 44th AIAA Aerospace Sciences Meeting, Jan. 2006, 2006-168.

${ }^{17}$ Boeuf, J. P., Lagmich, Y., Callegari, T., Pitchford, L. C., and Unfer, T., "New Insights in the Physics of DBD Plasma Actuators for Flow Control," 46th AIAA Aerospace Sciences Meeting, Jan. 2008, 2008-1376.

${ }^{18}$ Suzen, Y. B., Huang, P. G., Jacob, J. D., and Ashpis, D. E., "Numerical simulations of plasma based flow control applications," 35th Fluid Dynamics Conference, June 2005, 2005-4633.

${ }^{19}$ Santhanakrishnan, A., Jacob, J. D., and Suzen, Y. B., "Flow Control Using Plasma Actuators and Linear/Annular Plasma Synthetic Jet Actuators," 3rd AIAA Flow Control Conference, June 2006, 2006-3033. 